\title{
Dosage de l'activité lipasique et des acides gras libres du lait par titration automatique colorimétrique
}

\author{
par \\ P. CARTIER *, Y. CHILLIARD et Marie-Paule CHAZAL * \\ avec la collaboration technique de Jeanne FLECHET \\ Laboratoire de la lactation, I.N.R.A.-Theix - 63122 Ceyrat
}

\section{S u m m a r y}

DETERMINATION OF MILK LIPASE ACTIVITY AND MILK FREE FATTY ACID CONTENT USING A COLORIMETRIC AUTOMATIC TITRATION

The colorimetric automatic titration of free fatty acids (FFA) has been studied, with the Metrohm titration assembly, from Jellema's proposals (1981), to determine free fatty acids in raw milk using the BDI method. It has also been applied to the measurement of milk (lipoprotein) lipase activity.

1. Using standard solutions of palmitic acid, the assay was sensitive, precise, repeatable and rapid $(20$ to $45 \mathrm{sec}$. per measurement). During routine utilization, the rate of a series of measurements was limited by the number of successive determination that could be made without emptying the recipient. This number depended on the volume of each sample in relation to the volume of the titration vessel and to the amount of titrated FFA. Indeed, when this amount increased, turbidity occurred and disturbed the measurement. This occurred more quickly when the alkali was sodium hydroxide rather than potassium hydroxide and when the solvant was ether/ethanol (instead of heptan or butanol). It took place later with tetrabutyl ammonium hydroxide (TBAH) as titrant.

2. When applied to the determination of milk fat FFA (0.1 to $0.25 \mathrm{ml}$ fat obtained by the miniaturized BDI extraction method), the automatic titration gave results comparable to those obtained by manual titration using an accurate microburette. The butanol/TBAH system was the one that allowed the greatest number of successive determinations to be made without any turbidity.

*Institut Technique de l'Elevage Bovin. 
From a practical point of view, the precision of the automatic method depended on that of fat sampling with a pipette (whereas it was weighed in the manual method) especially with the miniaturized variants of the BDI method. Otherwise, its rapidity was limited by several precautions that had to be taken during calibration and during each series of determinations.

3. The lipoprotein lipase activity assay was based on the hydrolysis of a commercial triglyceride emulsion (Intralipid) containing long chain fatty acids, activated by bovine serum. Free fatty acids were extracted before and after enzymatic incubation by the method of Trout et al. (1960) and titrated on $3 \mathrm{ml}$ of heptan phase. Although the automatic method overestimated the absolute values of free fatty acids, lipase activities (development of FFA during incubation) measured by the 2 methods were equal and correlated very well. During routine utilization, the speed of the method was decreased strongly on account of the volume of each sample, because of titration vessel emptying and recalibration frequency.

4. Finally, the automatic titration did not seem to allow any time to be saved for lipoprotein lipase measurement. With the determination of milk FFA (extracted by the BDI method) time can be saved, in addition to inherent advantages linked to the suppression of the visual judgment of the change in colour (removal of operator effect and decreased visual weariness). Further studies are needed to evaluate the suitability of this kind of method for large-scale routine assays.

Keys words:

Milk - Fat-acidity - Lipase Activity - Free fatty acids - Colorimetric and automatic titration - Heptan - Butanol - Tetrabutyl ammonium hydroxide.

\section{R é s u m é}

Le dosage des acides gras libres (AGL) et de l'activité lipasique du lait par titration colorimétrique automatique a été étudié à l'aide de la chaîne de dosage Métrohm, à partir des propositions de Jellema (1981).

L'étalonnage de la méthode automatique montre qu'elle est sensible, précise, répétable et rapide. La rapidité d'une série de mesures est toutefois limitée par l'apparition d'un précipité, surtout lorsque le titrant est de la soude ou de la potasse et lorsque le solvant est le mélange éther/éthanol. L'emploi du tétra-butylammonium hydroxide comme titrant et du butanol ou de l'heptane comme solvant, permet de retarder l'apparition du précipité.

La méthode automatique est applicable au dosage de l'activité lipasique du lait, mais sans gain de temps par rapport à la méthode manuelle, en raison du volume des prises d'essai nécessaires au 
dosage. Par contre, l'automatisation de la titration des AGL du lait extraits par la méthode BDI peut permettre un gain de temps mais nécessite un pipettage volumétrique délicat de la matière grasse. Dans les 2 cas, l'automatisation permet de supprimer l'effet opérateur (appréciation visuelle du virage) et de diminuer la fatigue visuelle. L'intérêt de ce type de dosage en ateliers semi-industriels demande toutefois à être précisé.

\section{A. INTRODUCTION}

Un grand nombre de méthodes d'appréciation du taux d'acides gras libres (AGL) du lait et de son activité lipasique comporte une extraction des acides gras suivie d'une titration des fonctions acides à l'aide d'une solution alcaline, en présence d'un indicateur coloré.

Cette étape fastidieuse et subjective limite le nombre d'échantillons pouvant être traités quotidiennement, accroît l'incertitude du dosage et rend délicate la comparaison de résultats obtenus par des manipulateurs différents.

La détermination automatique du point d'équivalence de la titration pourrait alléger ce type de manipulation tout en permettant éventuellement d'augmenter la reproductibilité et la fiabilité des résultats. Les différences existant entre les spectres d'absorption de l'indicateur coloré en milieux acide et basique permettent la détection du point de virage par colorimétrie ; Jellema $(1979,1981)$ a proposé une méthode colorimétrique pour la titration automatique des AGL du lait.

Le but du présent travail est d'estimer l'intérêt de ce type de titration à l'aide de la chaîne de dosage Métrohm, à la fois pour le dosage de l'activité lipasique du lait et pour la détermination de sa teneur en acides gras libres.

\section{B. MATERIEL ET METHODES}

\section{Produits utilisés}

Tous les produits sont purs pour analyse, et utilisés tels qu'ils sont reçus :

- Heptane, isopropanol, éther diéthylique, butanol, éthanol $95 \%$, bleu de thymol (Merck) ;

- Tétra-n-butylammonium hydroxide (TBAH) en solution 0,1 M dans le mélange propanol - 2/ méthanol (Merck) ; labo) ;

- Soude, potasse, acides orthophosphorique et sulfurique (Pro- 
- Titron-X-100, hexamétaphosphate de sodium (Fluka);

- Albumine (fraction V), héparine (Sigma) ;

- Intralipide (Vitrum).

\section{$2^{\circ}$ Titration automatique}

a) Description du poste de titration

Le poste de titration (Métrohm) est constitué (figure 1) :

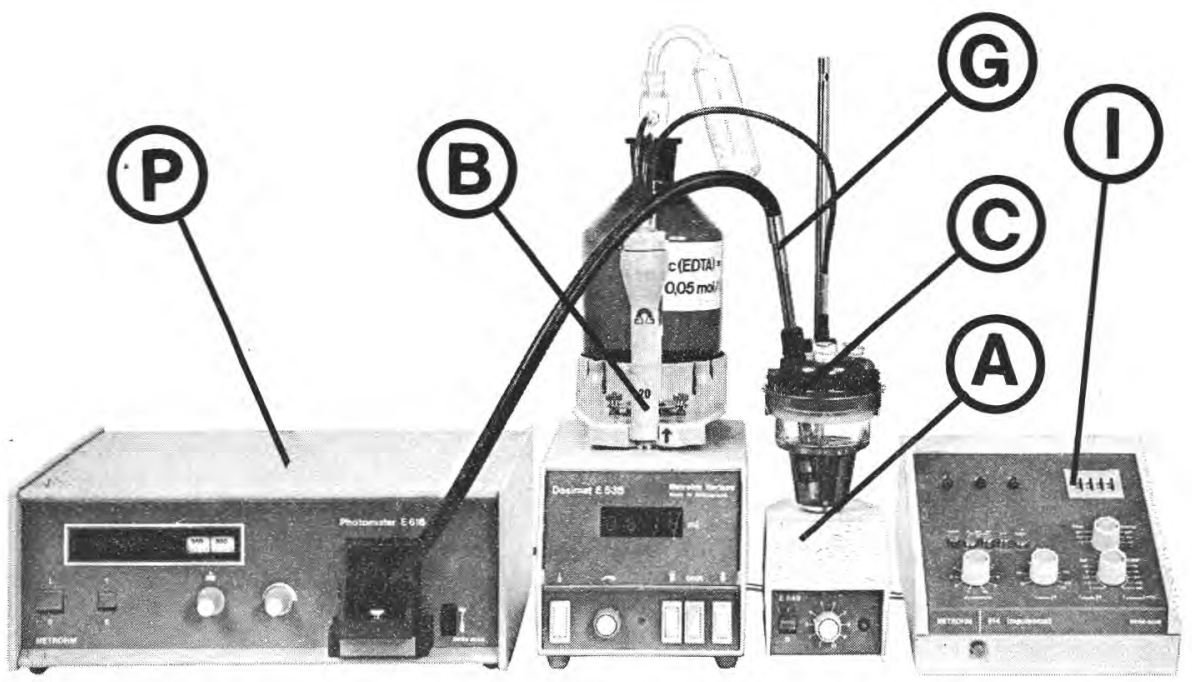

Figure 1

Poste de titration à point final et à indication colorimétrique (METROHM). A. Agitateur magnétique - B. Burette à piston motorisé - C. Cuve de mesure G. Guide optique - I. Régulateur à impulsions - P. Photomètre.

Colorimetric end-point titration assembly (METROHM).

A. Magnetic stirrer - B. Automatic burette - C. Recipient for measurements G. Optical conductor - I. Impulsomat - P. Photometer.

- d'un photomètre E 616 (P) possédant une gamme spectrale de 400 à $700 \mathrm{~nm}$, muni d'un guide optique $\mathrm{G}$ en fibre de verre relié à la cuve de mesure (C);

- d'une burette à piston motorisé (Multi-Dosimate 655) munie d'un piston de $1 \mathrm{ml}$;

— d'un régulateur à impulsions (Impulsomate 614) (I) ; 
- d'une cuve de mesure (C) en verre (capacité $80 \mathrm{ml}$ environ) surmontée d'un couvercle sur lequel s'adaptent l'extrémité du guide optique, la pointe de la burette (arrivée du titrant) et le tuyau pour le courant d'azote, et comportant un $4^{\circ}$ orifice par lequel peut être injecté l'échantillon à analyser. La cuve est située sur un agitateur magnétique (A).

b) Principe du FonctionNement : apPlication aU Dosage DES AGL PAR TITRATION A POINT FINAL

Cet appareil peut être utilisé pour la détermination du point d'équivalence des dosages titrimétriques à l'aide d'un indicateur coloré. Le photomètre émet un faisceau monochromatique conduit

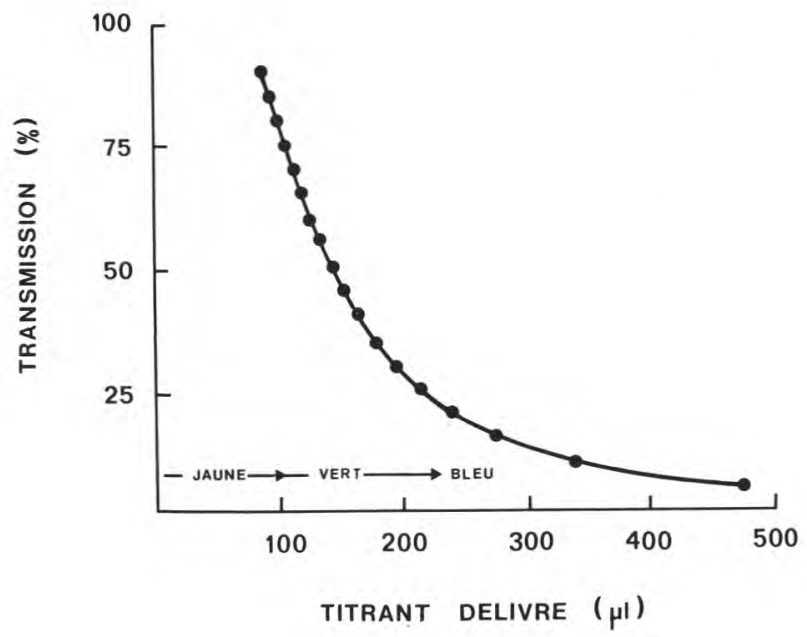

Figure 2

Evolution du pourcentage de transmission d'une solution de bleu de thymol au voisinage de la zone de virage.

- La cuve de mesure contient initialement: $16 \mathrm{ml}$ de butanol, $4 \mathrm{ml}$ de bleu de thymol à $1 \%$ dans l'isopropanol.

- $200 \mu \mathrm{l}$ d'une solution d'acide palmitique à $4,4 \mu$ eq $\mathrm{H}+/ \mathrm{ml}$ de butanol sont ajoutés.

- Solution titrante : TBAH dans l'isopropanol (environ $8.10^{-3} \mathrm{~N}$ ).

- Faisceau émis par le photomètre : $\lambda=610 \mathrm{~nm}$.

- $100 \%$ de transmission: jaune vif de l'indicateur en milieu acide.

Evolution of thymol blue transmittance near the end point.

The recipient initially contained:

$-16 \mathrm{ml}$ of butanol and $4 \mathrm{ml}$ of thymol blue (1 p. 1000 in isopropanol).

- $200 \mu \mathrm{l}$ of palmitic acid in butanol $(4.4 \mu \mathrm{Eq} \mathrm{H+} / \mathrm{ml})$ werre added.

- Working conditions: alkali: TBAH ( $\simeq 8.10^{-3} \mathrm{~N}$ in isopropanol).

- Wavelength: $\lambda=610 \mathrm{~nm}$.

- $100 \%$ transmittance: Yellow of the indicator in acidic medium. 
dans la cuve de mesure par une fibre de verre du guide optique. La longueur d'onde du faisceau est choisie de façon à ce que l'absorption soit minimale avant le virage (coloration de l'indicateur en milieu acide) et maximale lorsque celui-ci est dépassé (coloration de l'indicateur en milieu basique). Le faisceau, après avoir traversé le milieu, est réfléchi par un miroir situé à l'extrémité du guide optique puis ramené à l'appareil de mesure par une seconde fibre de verre. La solution titrante est délivrée automatiquement par la burette jusqu'à ce que le pourcentage de lumière retransmise au photomètre atteigne la valeur présélectionnée par l'opérateur (valeur de consigne du dosage). Cette valeur doit être choisie de façon à ce que les déterminations soient effectuées au virage de l'indicateur (cf. Figure 2), celui-ci devant être le plus proche possible du point d'équivalence du dosage.

\section{c) REMPlissage de LA CUVE ET RÉGLAge DE L'APPAREIL}

On place dans la cuve au minimum $20 \mathrm{ml}$ du mélange indicateur/ solvant (voir ci-dessous) et on adapte sur le couvercle les différents éléments. La pointe de la burette doit être dirigée vers le guide optique, le plus près possible, ce qui empêche dans une large mesure les dépassements du point de consigne. Il est préférable que l'extrémité du guide optique soit inclinée afin de limiter la formation de bulles sur le miroir. Par ailleurs, ce phénomène est restreint si l'agitation n'est pas excessive, et si le courant d'azote reste à la surface du solvant.

On définit ensuite les conditions de mesure en choisissant la longueur d'onde du faisceau émis et en présélectionnant le point de consigne sur l'impulsomate ; enfin, on règle le $100 \%$ de transmission sur la solution de bleu de thymol (indicateur coloré) dans le solvant d'étude (coloration jaune vif en milieu faiblement acide).

Pour notre étude, la plupart des dosages ont été réalisés dans les conditions suivantes :

- $\lambda$ émis : $610 \mathrm{~nm}$;

— point de consigne : 75 ou $70 \%$ (voir résultats) ;

- $\mathrm{t}$ delay: valeur minimale de l'appareil (1) ;

- dynamic : de 3 à 5 (1) ;

- $\mathrm{t}$ clock : 1,5 (1).

(1) Pour que les dosages soient relativement rapides sans pour autant dépasser le point de consigne, les impulsions doivent être de plus en plus brèves et éloignées lorsque l'on se rapproche de la valeur du point de consigne. Cette modulation de la libération du titrant est obtenue à partir des réglages effectués sur l'impulsomate: le réglage "dynamic» modifie la durée des ajouts de titrant, et le réglage " $t$ clock» la durée de l'intervalle entre impulsions, en fonction de l'écart entre le pourcentage de lumière transmise au photomètre et celui du point de consigne. Lorsque cet écart reste nul pendant une durée prédéterminée par le réglage " $\mathrm{t}$ delay », le dosage est terminé. 


\section{$3^{\circ}$ Activité lipoprotéine-lipasique ( $\left.L P L\right)$ du lait}

L'activité LPL du lait est appréciée comme décrit par Chilliard et Fehr (1976) en mesurant l'hydrolyse d'une émulsion commerciale d'Intralipide (contenant $10 \%$ d'huile de soja c'est-à-dire de triglycérides à acides gras à longue chaîne) en présence de sérum sanguin, d'albumine et d'héparine, pendant 30 minutes.

Les acides gras libres initiaux $(\mathrm{T}=0)$ ou après incubation $(\mathrm{T}=30 \mathrm{mn})$ et les solutions étalon d'acide palmitique sont extraits par le mélange isopropanol-heptane-H2 $\mathrm{SO} 4$ selon la méthode de Trout et al. (1960), qui s'est avérée quantitative et spécifique (Chilliard et al., 1984).

La teneur en AGL de la phase heptanoïque est déterminée sur $3 \mathrm{ml}$. Pour des titrations manuelles, on utilise une burette de précision (Métrohm E 457) équipée d'un piston de capacité 0,5 $\mathrm{ml}$. Les dosages sont effectués par de la soude $\left(10^{-2} \mathrm{M}\right.$ dans l'isopropanol), en présence de 6 gouttes de bleu de thymol $(0,1 \%$ dans l'isopropanol) et sous bullage d'azote afin d'éviter l'interférence du gaz carbonique. Les titrations automatiques sont réalisées en introduisant la phase heptanoïque dans la cuve de mesure qui contient initialement $4 \mathrm{ml}$ de solution de bleu de thymol et $16 \mathrm{ml}$ d'heptane. Un courant d'azote est maintenu à la surface du solvant.

\section{$4^{\circ}$ Dosage des AGL du lait par la méthode BDI miniaturisée}

Le dosage est effectué selon la méthode BDI (Bureau of Dairy Industry, Thomas et al., 1955) modifiée d'après Driessen et al. (1977) et Hänni et Rychener (1980). Cette méthode a été choisie car elle est universellement utilisée, très spécifique, répétable et reproductible (Chilliard et al., 1983). De plus, le dosage direct sur la matière grasse peut permettre une automatisation plus poussée (Driessen et al., 1977 ; Jellema, 1979, 1981).

La matière grasse est extraite sous l'action d'une solution détergente (hexamétaphosphate de sodium: $70 \mathrm{~g} / 1$; Triton X-100: $30 \mathrm{~g} / 1$; $\mathrm{pH}$ ajusté entre 5 et 5,5 à l'aide d'acide orthophosphorique pur) à raison de $4,5 \mathrm{ml}$ pour $16 \mathrm{ml}$ de lait (méthode miniaturisée). Après chauffage à $100^{\circ} \mathrm{C}$ pendant 20 minutes, les échantillons sont refroidis à $45^{\circ} \mathrm{C}$ puis un aliquot de la matière grasse surnageante est prélevé afin d'être titré.

Lorsque les titrations sont effectuées manuellement, la matière grasse est prélevée à l'aide d'une pipette Pasteur à large embout, introduite dans des tubes à col rodé de $15 \mathrm{ml}$, pesée (au mg près) puis solubilisée dans $5 \mathrm{ml}$ de mélange éther diéthylique/éthanol 50/50 (V/V) et titrée de façon similaire à celle décrite pour l'activité LPL du lait (la burette étant alors équipée d'un piston de $5 \mathrm{ml}$ ).

Lorsqu'on utilise le poste de titration, 50 à $200 \mu \mathrm{l}$ de phase grasse surnageante (prélevés précisément avec une pipette de $200 \mu \mathrm{l}$ ) sont 
directement introduits dans la cuve de mesure qui contient initialement $32 \mathrm{ml}$ de solvant et $8 \mathrm{ml}$ de la solution de bleu de thymol $(0,1 \%$ dans l'isopropanol).

Dans les 2 cas, on procède au dosage d'une gamme étalon d'acide palmitique.

\section{RESULTATS ET DISCUSSION}

\section{Etalonnage de la titration automatique}

L'étalonnage de la titration (choix de la valeur de consigne, comparaison de 3 solvants et de 3 titrants) a été réalisé en dosant des aliquots de $1 \mathrm{ml}$ de solutions d'acide palmitique de titre connu ( 2 ou $3 \mu \mathrm{eq} / \mathrm{ml}$ ).

Lorsque le solvant utilisé est l'heptane, l'emploi de soude ou de potasse comme titrants conduisent à l'apparition d'un précipité dans la cuve dès le $4^{\circ}$ ou le $20^{\circ}$ ajout respectivement. Par contre, aucun précipité n'a été observé lors de l'utilisation de TBAH pour au moins 30 dosages successifs effectués dans le butanol ou dans l'heptane. En ce qui concerne les dosages effectués dans le mélange éther/ éthanol, le nombre de mesures pouvant être effectuées dans une même série (2) est identique en présence de soude ou de TBAH (une quinzaine de dosages environ) mais reste inférieur à celui obtenu avec le TBAH et les 2 solvants précédents.

La répétabilité de la méthode a été estimée en utilisant les 3 solvants, et le TBAH comme titrant. Les résultats (tableau I) montrent que les coefficients de variation des manipulations effectuées dans l'heptane et dans le butanol sont relativement faibles (de l'ordre de $2 \%$ ). Par contre, une plus grande dispersion peut apparaître lors de l'emploi du mélange éther-éthanol comme solvant, mais pas systématiquement. Outre une concentration des solutions étalon, ce fait peut être dû à l'instabilité de la coloration au voisinage du point de consigne, également observée par Jellema (1979) et attribuée à l'évaporisation de l'éther. Ce phénomène augmente la durée de chaque dosage mais semble toutefois être limité avec la concentration en bleu de thymol employée dans cette étude, qui est 10 fois supérieure à celle utilisée par Jellema (1981).

Les acidités mesurées semblent légèrement sous-estimées pour un point de consigne à $75 \%$ de transmission; par contre la présélection à $70 \%$ de transmission permet de se trouver au voisinage du point d'équivalence du dosage comme en témoigne le rapport " $\mathrm{OH}^{-}$versé/ $\mathrm{H}^{+}$apporté » (tableau 1). Par ailleurs, cètte valeur correspond au point de virage de l'indicateur (cf. figure 2) de telle sorte

(2) On entend par série de mesures l'ensemble des dosages effectués dans la cuve de mesure sans la vider. 


\section{TABLEAU 1 - TABLE 1}

Titration automatique de solutions d'acide palmitique dans 3 solvants différents: étalonnage et répétabilité de la méthode.

Automatic titration of standard solutions of palmitic acid using three different solvents: standardization and repeatability of the method.

\begin{tabular}{|c|c|c|c|}
\hline Solvant & Heptane & Butanol & Ether/Ethanol \\
\hline Titrant & & $\leftarrow$ ТВАН $10^{-2} \mathrm{M}$ & $\rightarrow$ \\
\hline Point de consigne & $75 \%$ & $70 \%$ & $70 \%$ \\
\hline Nombre de titrations & $\mathrm{n}=38$ & $\mathrm{n}=8$ & $\mathrm{n}=10$ \\
\hline ueq AGL par ajout (1) & 1,95 & 2,93 & 2,93 \\
\hline $\begin{array}{l}\mu l \text { de TBAH délivré (5) } \\
\qquad(\overline{\mathrm{x}} \pm \mathrm{sx})\end{array}$ & $188 \pm 4$ & $288 \pm 8$ & $307 \pm 20$ \\
\hline Coefficient de variation & $2 \%$ & $3 \%(3)$ & $6,5 \%(4)$ \\
\hline $\mathrm{OH}^{-}$versé $/ \mathrm{H}^{+}$apporté & 0,96 & 0,98 & 1,05 \\
\hline
\end{tabular}

(1) On procède à des ajouts d'aliquots de $1 \mathrm{ml}$ de solution d'acide palmitique à $1,95 \mu \mathrm{eq} / \mathrm{ml}$ (étude dans l'heptane) ou $2,93 \mu \mathrm{eq} / \mathrm{ml}$ (étude dans le butanol ou l'éther-éthanol).

(2) Coefficient de variation: 1,3 à $2,3 \%$ pour 3 autres séries $(\mathrm{n}=45)$.

(3) Coefficient de variation : $1,8 \%$ pour 1 autre série $(n=13)$.

(4) Coefficient de variation: $1,4 \%$ pour 1 autre série $(n=5)$.

(5) De ces valeurs ont été déduit les blancs (titration de $1 \mathrm{ml}$ de solvant sans acide palmitique) soit environ $3 \mu \mathrm{l}$ de TBAH $10^{-2} \mathrm{M}$.

(1) One $\mathrm{ml}$ of palmitic acid solution $(1.95 \mu \mathrm{Eq} / \mathrm{ml}$ heptan or $2.93 \mu \mathrm{\mu q} / \mathrm{ml}$ other solvents) were transferred to the recipient.

(2) Coefficient of variation: 1.3 to $2.3 \%$ for 3 other series of measurements $(n=45)$.

(3) Coefficient of variation: $1.8 \%$ for another series of measurements $(n=13)$.

(4) Coefficient of variation: $1.4 \%$ for another series of measurements $(n=5)$.

(5) The acidity of the solvent alone was subtracted (about $3 \mu \mathrm{l}$ of $10^{-2} \mathrm{M}$ $T B A H)$.

que la méthode est très sensible. Ainsi, le virage du bleu de thymol permet d'apprécier très précisément le point d'équivalence des dosages d'AGL en milieu non aqueux, conformément aux résultats obtenus par Driessen et al. (1977) dans une comparaison avec une méthode potentiométrique. 
2. Dosage de l'activité lipoprotéine-lipasique du lait: titration automatique des acides gras libres dans l'heptane - Comparaison avec la méthode manuelle

L'activité lipasique de 29 laits a été mesurée.

La comparaison des teneurs en AGL appréciées manuellement ou automatiquement (figure 3) montre une bonne corrélation entre

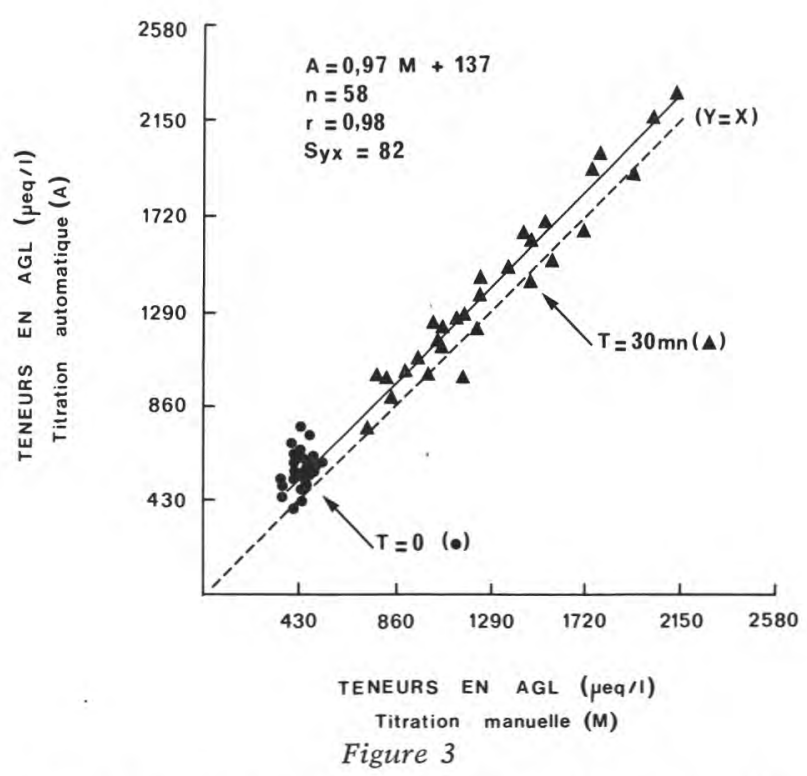

Dosage de l'activité lipoprotéine lipasique du lait: Teneurs en AGL du milieu avant $(T=0)$ et après incubation $(T=30)$, estimées par titration manuelle et par titration automatique, après extraction par la méthode de Trout et al. (1960).

Determination of lipoprotein lipase activity in raw milk: amount of free fatty acids before $(T=0)$ and after $(T=30)$ incubation, estimated by automatic $(A)$ and manual (M) titrations (FFA were extracted by the method of Trout and al., 1960).

les deux types de titration. Cependant, les résultats obtenus par titration automatique sont plus élevés (d'environ $140 \mu \mathrm{Eq} / \mathrm{l}$ ) sur toute la plage des valeurs étudiées. En outre, les valeurs initiales $(T=0)$ sont plus dispersées lorsqu'elles sont mesurées automatiquement que manuellement (figure 3). Des études supplémentaires sont nécessaires pour expliquer cette surestimation d'autant plus que celle-ci serait gênante pour déterminer les valeurs absolues des teneurs en AGL de milieux biologiques (plasma par exemple), but initial de la méthode de Trout et al. (1960). 
Les activités LPL (proportionnelles à la différence $\mathrm{AGL}_{\mathrm{T}=30}$ $\mathrm{AGL}_{\mathrm{T}=0}$ ) obtenues manuellement et automatiquement sont très voisines (figure 4), en raison du décalage constant (à $\mathrm{T}=0$ et $\mathrm{T}=30$ ) entre les deux modes de titration (figure 3 ). L'écart type résiduel est faible $(9,6 \mu \mathrm{eq} / \mathrm{h} / \mathrm{ml})$, soit un coefficient de variation résiduelle de $11 \%$.

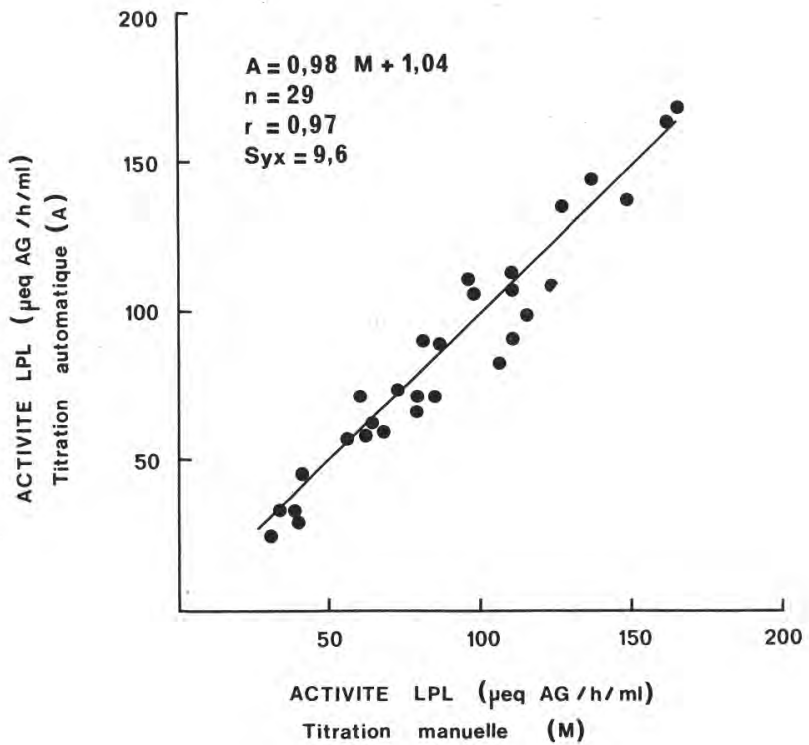

Figure 4

Comparaison des activités lipoprotéine-lipasiques de plusieurs laits, obtenues par titration manuelle (M) ou automatique (A) des acides gras libérés pendant l'incubation. L'activité LPL est calculée à partir des teneurs en AGL du milieu avant et après incubation, représentées figure 3.

Relationship between automatic $(A)$ and manual $(M)$ titrations in the assay of milk LPL activity. LPL activity is calculated according to the amount of FFA that were liberated during incubation (See figure 3).

Cependant, l'automatisation de la titration ne permet aucun gain de temps. En effet, le volume d'extraction de chaque échantillon étant relativement important $(3 \mathrm{ml})$, le nombre de dosages effectués par série est restreint (de l'ordre de 15 avec la cuve utilisée, de $80 \mathrm{ml}$ ), si bien qu'un plus grand nombre de séries de titrations est nécessaire. Ceci augmente considérablement le temps de manipulation, en multipliant les opérations de remplissage et de réglage précédant chaque série. Ce problème pourrait être résolu en diminuant le volume de chaque échantillon et en utilisant une cuve de mesure de plus grande 
capacité. Cependant, la première solution n'est guère envisageable compte tenu des faibles teneurs en AGL des échantillons. La seconde, quant à elle, ne semble pas sans inconvénient (variation de la concentration en indicateur coloré, modification du réglage de l'agitation, augmentation du temps de stabilisation de la coloration avec l'augmentation du volume) et nécessiterait d'être testée avant de pouvoir être adoptée.

\section{Titration automatique de l'acidité de la matière grasse du lait extraite par la méthode BDI - Comparaison avec les valeurs obtenues par titration manuelle}

\section{a) ETUdES PRÉLIMINAIRES}

Nous avons vérifié que le volume de titrant délivré reste proportionnel à la quantité de matière grasse introduite dans la cuve (de 50 à $1000 \mu \mathrm{l}$ au moins), ce qui rend possible les déterminations à partir de quantités variables de matière grasse. Par ailleurs, il est préférable d'utiliser le butanol comme solvant et d'effectuer les titrations avec du TBAH car avec ce système aucun précipité n'apparaît avant au moins 40 titrations utilisant chacune 100 à $200 \mu l$ de matière grasse (provenant de laits contenant en moyenne $2000 \mu$ eq AGL/100 g de matière grasse), contrairement à ce qui est observé avec le mélange éther-éthanol comme solvant.

b) COMPARAISON DES TENEURS EN AGL DE DIFFÉRENTS LAITS, DÉTERMINÉES SELON LA MÉTHODE BDI, PAR TITRATION MANUELLE OU AUTOMATIQUE

Deux manipulations ont été effectuées, respectivement à partir de 10 et 6 laits présentant des teneurs en AGL comprises entre 200 et 7500 ueq/100 g de matière grasse.

Sur l'ensemble des deux manipulations, l'équation de régression liant les titrations automatiques $(\mathrm{A})$ aux titrations manuelles $(\mathrm{M})$ est : $\mathrm{A}=0,97 \mathrm{M}-116$ (figure 5). Les deux manipulations conduisent respectivement aux droites de régression suivantes : $\mathrm{A}=0,94 \mathrm{M}-171$ (a) et $A=1,04 M-34(\mathbf{\Delta})$. L'écart type poolé intra série (3) de la titration automatique $(120 \mu \mathrm{eq} / 100 \mathrm{~g}$ de matière grasse) reste supérieur à celui de la titration manuelle ( $60 \mu \mathrm{eq} / 100 \mathrm{~g}$ de matière grasse).

Dans les conditions de cet essai, la liaison entre les titrations manuelles et automatiques effectuées simultanément sur une quinzaine d'échantillons de laits est bonne. Cependant, un des facteurs limitant la précision de la méthode automatique semble résider dans le pipettage de la matière grasse, qui reste visqueuse à $45^{\circ} \mathrm{C}$, alors qu'avec la méthode manuelle la pesée permet une prise d'essai très précise.

(3) Calcul effectué à partir des écarts entre les doubles réalisés pour un même lait par la formule $: \sigma=\left(\Sigma \mathrm{di}^{2} / 2 \mathrm{n}\right) 1 / 2$. 


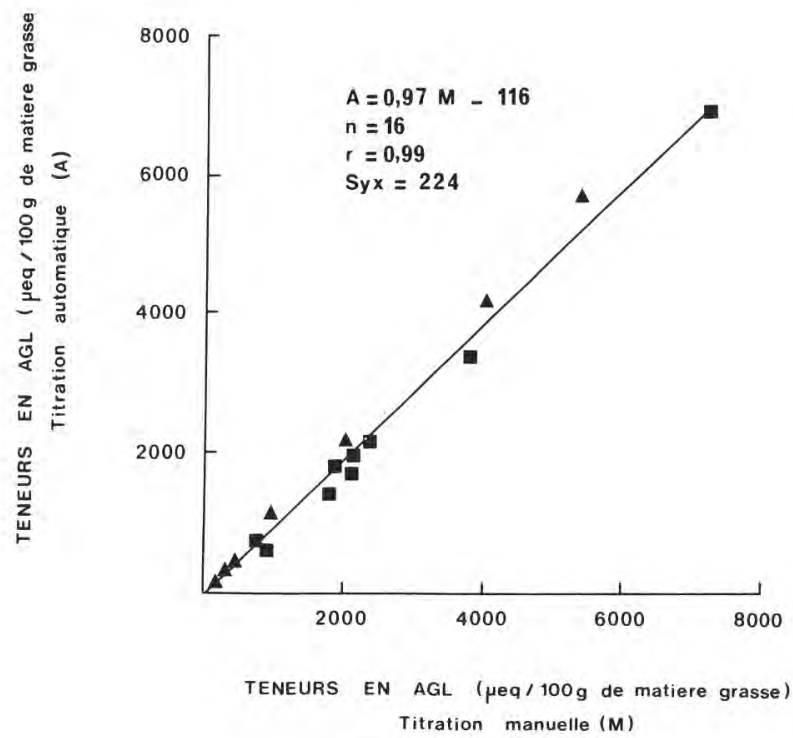

Figure 5

Teneurs en AGL de différents laits déterminées par la méthode BDI et par titration manuelle $(\mathrm{M})$ ou automatique $(\mathrm{A})$. Les résultats de 2 séries ( $\mathbf{\Delta}$ - $)$ ont été regroupés. Chaque point représente un lait différent. Les valeurs de titration automatique représentent une détermination ( $\mathbf{\Delta}$ ) ou la moyenne de 2 à 4 déterminations (a). Les valeurs de titration manuelle sont la moyenne de 2 déterminations.

Determination of milk fat acidity (acid degree value) by the BDI method: comparison of manual $(M)$ and automatic $(A)$ titrations. Results from 2 series of measurements ( $\mathbf{\Delta}, \mathbf{)}$ ) were used. Each point corresponds to one milk sample. Automatic titrations were obtained from one measurement (A), or 2 to 4 determinations per milk sample ( $\mathbf{(})$. Manual titrations were the means of two determinations.

Par ailleurs, en routine, la miniaturisation de la méthode BDI (extraction à partir de $16 \mathrm{ml}$ de lait, soit en moyenne $650 \mathrm{mg}$ de matière grasse) rend délicat le pipettage d'une ou plusieurs parties aliquotes de $200 \mu \mathrm{l}$ dans la phase grasse surnageante en utilisant des tubes à essai classiques et avec des laits pauvres en matière grasse. Ce problème peut être résolu en utilisant des tubes à col étroit (Driessen et al., 1977 ; Chazal et al., 1984).

Contrairement à la titration automatique des extraits heptanoïques d'AGL lors du dosage de l'activité lipoprotéine-lipasique du lait, il est possible d'effectuer un grand nombre d'ajouts de 50 à $200 \mu \mathrm{l}$ de matière grasse dans la cuve de mesure sans vider celle-ci. Cependant le gain de temps semble être limité du fait des précautions qui doivent être prises pour le pipettage de la matière grasse et tout au long de la mesure. Ces précautions ont notamment pour but 
d'éviter la formation de bulles (par un réglage correct de l'agitation et du bullage d'azote lorsque varie le volume de solvant), de maintenir une position correcte du diffuseur de titrant par rapport à la sonde photométrique, d'adapter la cinétique de libération du titrant si les déterminations deviennent trop longues ou lorsque le point de consigne est dépassé.

\section{CONCLUSION}

Cette étude confirme les résultats de Jellema $(1979,1981)$ montrant que la titration automatique colorimétrique à point final peut être mise à profit pour la détermination des teneurs en acides gras libres en milieu non aqueux. Nous avons essayé cette méthode pour deux types de mesure : le dosage des acides gras longs libérés par la lipase du lait in vitro à partir d'une émulsion d'Intralipide puis extraits en phase heptanoïque, et celui des AGL contenus dans la matière grasse du lait extraite selon la méthode BDI.

L'introduction de la méthode automatique ne semble pas permettre d'accélérer fortement les cadences de travail, bien qu'individuellement chaque dosage soit relativement rapide (de 20 à 45 secondes par détermination). Avec la méthode manuelle, un opérateur entraîné peut réaliser chaque semaine 3 séries d'environ 100 dosages d'AGL par la méthode de Trout et al. (1960) ou trois séries de 40 déterminations d'activité lipasique (compte tenu du temps nécessaire pour préparer et réaliser l'incubation enzymatique). Par ailleurs, il est possible de réaliser hebdomadairement 3 à 4 séries de 70 à 90 dosages d'AGL du lait par la méthode BDI manuelle miniaturisée.

Le principal avantage de l'automatisation semble être la suppression de l'appréciation visuelle du point de virage, permettant une meilleure reproductibilité entre opérateurs. Par ailleurs, en rendant le dosage moins pénible, elle pourrait permettre une augmentation du nombre de séries réalisables hebdomadairement par un opérateur spécialisé sur ce type de dosage. Des études supplémentaires sont nécessaires pour apprécier l'intérêt de cette automatisation en ateliers semi-industriels.

Les conditions opératoires retenues à l'issue de cette étude pour la méthode BDI sont résumées dans le tableau 2.

\section{Remerciements}

Nous remercions la Société Roucaire (B.P. 65, F 78143 Velizy-Villacoublay) pour avoir mis à notre disposition temporairement la chaîne de dosage Métrohm, et notamment MM. Jallade et Million pour leurs conseils lors de la mise en route de l'appareit.

Cette étude a été réalisée grâce au concours financier du Ministère de l'Industrie et de la Recherche, et de l'Institut Technique de l'Elevage Bovin. 


\section{TABLEAU 2 - TABLE 2}

Dosage des AGL du lait par titration colorimétrique à partir de la matière grasse extraite selon la méthode BDI miniaturisée : récapitulation des conditions de mesure dans le cas d'une automatisation de la titration (titration colorimétrique à point final).

Determination of milk fat acidity $(A D V)$ by the miniaturized BDI extraction method: summary of working conditions when the titration was automated (colorimetric end-point titration).

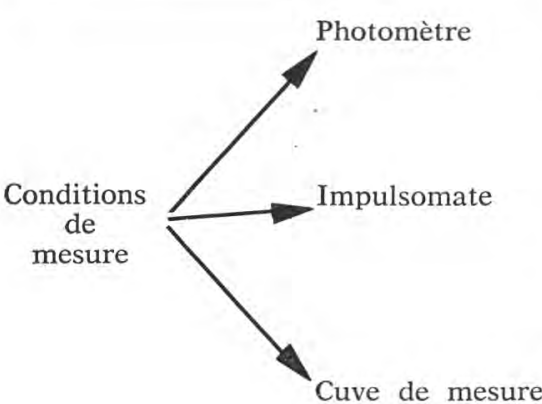

Cuve de mesure
- Travail en transmission

- Longueur d'onde émise : $\lambda=610 \mathrm{~mm}$

- Point de consigne: $70 \%$

- Caractéristiques de libération du titrant :

- dynamic: $3 \rightarrow 5$

- $\mathrm{t}$ clock: 1,5

- Arrêt de la mesure - $t$ delay minimum

- Sonde du titrant au contact du guide optique

- Bullage d'azote en surface

- Agitation magnétique minimale

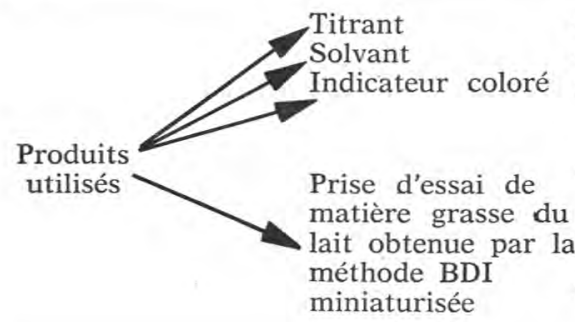

- TBAH $10^{-2} \mathrm{M}$ dans l'isopropanol

- Butanol-1 : $32 \mathrm{ml}$

- Bleu de thymol: $8 \mathrm{ml}$ (solution à $1 \%$ dans l'isopropanol)

- Mode de prélèvement: pipettage volumétrique avec une pipette automatique calibrée de $200 \mu \mathrm{l}$

- Volume de 50 à $200 \mu \mathrm{l}(\mathrm{d}=0,94)$

- Nombre de dosages: au moins 40 par série de mesures.

\section{Références bibliographiques}

Chazal (M. P.), Cartier (P.), Chilliard (Y.), Fléchet (J.), Bauchart (D.), Duboisset (F.), Meyer (M.) (1984). - Dosage des acides gras libres du lait par la méthode BDI. Cahier des Techniques de l'INRA, $\mathrm{n}^{\circ} 6$, sous presse.

Chilliard (Y.), Bauchart (D.), Barnouin (J.) (1984). - Determination of plasma non-esterified fatty acids in herbivores and man: a comparison of values obtained by manual or automatic chromatographic, titrimetric, colorimetric and enzymatic methods. Reprod. Nutr. Dévelop., 24 (4), sous presse.

Chilliard (Y.), Bauchart (D.), Cartier (P.) (1983), - Comparaison de 5 méthodes titrimétriques avec une méthode chromatographique de dosage des acides gras libres du lait de vache. Brochure ITEB-INRA, $n^{\circ} 84031$.

Chilliard (Y.), Fehr (P.M.) (1976). - Activité lipolytique du lait de chèvre. I. Mise en évidence d'une activité lipoprotéine-lipasique. Ann. Technol. agric., $25,219-230$. 
Driessen (F. M.), Jellema (A.), Van Luin (F. J. P.), Stadhouders (J.), WolBERS (G. J. M.) (1977). - The estimation of the fat acidity in raw milk. An adaptation of the BDI method, suitable for routine assays. Neth. Milk Dairy J., 31, 40-55.

HANnI (H.), RychenER (M.) (1980). - Vergleich verschiedener Methoden zur Bestimmung der freien Fettsäuren in Milch und Milchprodukten. Mitt. Gebiete Lebensm. Hyg., 71, 509-525.

Jellema (A.) (1979). - Titration automatique à l'aide d'un colorimètre pour la détermination de l'acidité de la matière grasse. (Document non publié communiqué au Comité Lipolyse de l'Association Laitière Française.)

Jellema (A.) (1981). - Determination of free fatty acids. Routine method using fat separation and automatic titration. Proposal for an I.D.F. standard (Document communiqué au Comité Lipolyse de l'Association Laitière Française).

Thomas (E. L.), Nielsen (A. J.), Olson (J.C.) (1955). - Hydrolytic rancidity in milk. A simplified method for estimating the extent of its development. Am. Milk Rev., 17, 50-51-85.

Trout (D. L.), Estes (E. H.), Friedberg (S. J.) (1960). - Titration of free fatty acids of plasma: a study of current methods and a new modification. J. Lipid. Res., 1, 199-202. 